\title{
Plasma neutrophil gelatinase-associated lipocalin as an early biomarker for prediction of acute kidney injury after cardio-pulmonary bypass in pediatric cardiac surgery
}

\author{
Fatina I. Fadel', Azza M.O. Abdel Rahman², Mohamed Farouk Mohamed', Sonia A. Habib², \\ Mona H. Ibrahim³, Zeinab S. Sleem¹, Hafez M. Bazaraa', Mohamed M.A. Soliman²
}

1Department of Pediatrics, Cairo University, Egypt

2Department of Pediatrics, National Research Centre, Egypt

${ }^{3}$ Department of Clinical Pathology, National Research Centre, Egypt

Submitted: 27 July 2011

Accepted: 23 September 2011

Arch Med Sci 2012; 8, 2: 250-255

DOI: $10.5114 /$ aoms.2012.28552

Copyright $\odot 2012$ Termedia \& Banach

\begin{abstract}
Introduction: Cardiopulmonary bypass (CPB) surgery is considered one of the most frequent surgical procedures in which acute kidney injury (AKI) represents a frequent and serious complication. The aim of the present study was to evaluate the efficiency of neutrophil gelatinase-associated lipocalin (NGAL) as an early AKI biomarker after CPB in pediatric cardiac surgery.

Material and methods: The study included forty children aged 2 to 78 months undergoing CPB. They were divided into group I: patients who suffered AKI grades II and III; and group II: patients who did not develop AKI or at risk. Peripheral venous blood was withdrawn pre- and post-operatively for serial measurements of NGAL and creatinine. Statistical analysis was performed using Statistical Package for Social Sciences version 14.

Results: Mean plasma NGAL levels showed highly significant elevations in group I patients at 2,12 , and $24 \mathrm{~h}$ after surgery $(p<0.0001)$ compared to group II. Significant correlations were found between NGAL and creatinine at different time intervals. Highly significant correlations $(p<0.0001)$ were found between plasma NGAL and AKI at 2, 12 and $24 \mathrm{~h}$ after surgery. A cut-off level of $100 \mathrm{ng} / \mathrm{ml}$ at $2 \mathrm{~h}$, and $125 \mathrm{ng} / \mathrm{ml}$ at $12 \mathrm{~h}$ post-operatively both recorded the highest accuracy, being $95 \%$ accurate, with sensitivity of $100 \%$ and $89.5 \%$ respectively, and specificity of $90.5 \%$ and $100 \%$ respectively.

Conclusions: This study showed that plasma NGAL could be used as an early biomarker for detection of AKI following CPB. We recommend further studies on a wider scale to validate the current study results.
\end{abstract}

Key words: neutrophil gelatinase-associated lipocalin, biomarker, acute kidney injury, cardio-pulmonary bypass, pediatrics.

\section{Introduction}

Acute kidney injury (AKI) is a term proposed to reflect the entire spectrum of acute renal failure (ARF), a complex disorder that occurs in a wide variety of settings with clinical manifestations ranging from minimal elevation in serum creatinine to anuric renal failure [1]. In 2004, a consensus definition for AKI was proposed by the Acute Dialysis Quality Initiative: the RIFLE criteria (risk, injury, failure, loss, end-stage renal disease) [2]. The adult-derived RIFLE definition was modified, and then applied and vali-

\author{
Corresponding author: \\ Dr. Azza M.O. Abdel Rahman \\ Department of Pediatrics \\ National Research Centre \\ Dokki, Egypt \\ Phone: +202 26366932 \\ E-mail : \\ azzaomar_74@yahoo.com
}


dated in pediatric patients and renamed as the pediatric RIFLE (pRIFLE) criteria. Pediatric RIFLE stratifies AKI from mild (RIFLE: R, risk) to severe (RIFLE: F, failure) based on changes in serum creatinine or estimated creatinine clearance $(\mathrm{eCCl})$ and urine output. The first study that defined AKI using the pRIFLE criteria found that AKI occurred in $82 \%$ of the most critically ill children admitted to a pediatric intensive care unit (PICU) [3].

The AKI represents a significant problem in clinical medicine, with devastating immediate and long-term consequences [4, 5]. The incidence of AKI varies from $5 \%$ of hospitalized patients to $30 \%$ of patients in intensive care units. Cardiopulmonary bypass (CPB) surgery is considered one of the most frequent major surgical procedures performed worldwide [6], in which AKI represents a frequent and serious complication in about $30-50 \%$ of patients [7]. The AKI requiring dialysis occurs in up to $5 \%$ of these cases, in whom the mortality rate approaches $80 \%$ [8].

Diagnosis and management of AKI constitutes one of the most important roles of the pediatric nephrologist in an intensive care unit (ICU) [9].

In current practice, AKI is typically diagnosed by measuring serum creatinine, which is a reliable marker of kidney function. Unfortunately, its constant and slow production makes its rise small and late in the case of acute injury. There remains a need for a much earlier marker in order to diagnose AKI as soon as possible [10].

Neutrophil gelatinase-associated lipocalin (NGAL), a $25 \mathrm{kDa}$ protein, is generally expressed in very low concentrations in several human tissues, and is greatly elevated in the case of epithelial damage $[11,12]$. In healthy kidneys, it is barely detectable in either plasma or urine. However, in the setting of acute tubular injury, NGAL undergoes rapid and profound up-regulation with large increases in both urine and plasma [13].

There are many ongoing studies to elucidate the nature of the association between NGAL and AKI in different critical care settings. This includes different types of cardiac surgery, contrast-induced nephropathy, worsening renal function in patients who suffer heart failure and sepsis.

The aim of the present study was to evaluate the efficiency of NGAL plasma level as an early AKI biomarker after CPB in pediatric cardiac surgery.

\section{Material and methods}

The present study was carried out in the cardiothoracic ICU, Children's Hospital, Cairo University and the Clinical Pathology Department of the National Research Centre (NRC) during the period from January $1^{\text {st }}, 2010$ to June $1^{\text {st }}, 2010$.

Forty children aged 2 to 78 months were enrolled in the study. They underwent CPB for variable peri- ods ranging from 10 up to 180 min depending on the type of anomaly and procedure done, which included surgical correction or palliation of congenital heart disease. Patients with pre-existing renal insufficiency, or suffering from peripheral vascular diseases, or on nephrotoxic drugs before surgery, as well as known diabetics were excluded from the study. The development of acute kidney injury was assessed using the pediatric RIFLE classification [2] (AKI grade I equivalent to RIFLE: risk, AKI grade II equivalent to RIFLE: injury, AKI grade III equivalent to RIFLE: failure). Informed consent from the patients' caregivers was obtained and approved by the Ethical Committee of the NRC and Cairo University Children's Hospital.

Patients included in the study were subjected to full history taking and thorough clinical examination focusing on blood pressure, central venous pressure, urine output, and respiratory and ventilatory support by assessment of fraction of inspired oxygen and mean airway pressure recorded before surgery and at 2, 12, and $24 \mathrm{~h}$ following surgery.

Laboratory investigations included complete blood count (CBC) including white blood cell and differential counts using ADVIA 60 (Bayer Diagnostics, UK); assessment of renal functions by estimation of serum levels of urea, creatinine, sodium, potassium, calcium and phosphorus; serum albumin level (Synchron CX5 automated chemistry analyzer); blood gas analysis using Nova 9 (Nova Biomedical, USA); blood cultures and sensitivity using Bactec 9050 (Becton Dickenson Diagnostic System, USA); plasma NGAL levels using Human Lipocalin-2/NGAL ELISA (BioVendor Research and Diagnostic Products, Czech Republic). Blood cultures and sensitivity tests were done for selected patients as clinically indicated at any time of the study depending on progression of the condition. Serum urea, creatinine, sodium, potassium and plasma NGAL levels were assessed preoperatively as well as at 2, 12 and $24 \mathrm{~h}$ following surgery.

\section{Sampling}

Preoperatively: $6 \mathrm{ml}$ of peripheral venous blood was withdrawn from every patient under complete aseptic conditions, $3 \mathrm{ml}$ of the withdrawn blood was anticoagulated with EDTA for performing quantitative measures of NGAL, and the rest was left to clot at room temperature, then centrifuged and sera were separated for assessment of urea, creatinine, sodium, potassium, calcium, phosphorus and albumin.

Postoperatively: Monitoring of the patients was accomplished by serial assessments of CBC, serum urea, creatinine, sodium, potassium and plasma $\mathrm{NGAL}$; and $3 \mathrm{ml}$ of arterial blood was withdrawn from every patient through an inserted arterial cannula and was added to heparinized tubes for assessment of arterial blood gases as well as acid base status. 
Table I. Demographic data of studied children

\begin{tabular}{|lc|}
\hline Variable & Patients $(n=40)$ \\
\hline Age (mean \pm SD) [months] & $20.75 \pm 23.33$ \\
\hline Sex (M/F) & $20 / 20$ \\
\hline Clinical diagnosis & 40 \\
\hline Large VSD [\%] & 15 \\
\hline TGA with VSD-ASD [\%] & 12.5 \\
\hline VSD-DORV [\%] & 10 \\
\hline ASD [\%] & 22.5 \\
\hline Others ${ }^{\dagger}[\%]$ & $4(10)$ \\
\hline Dialysis requirement, $n(\%)^{*}$ & $9(22.5)$ \\
\hline Non-Survivors, $n\left(\%{ }^{* *}\right.$ & \\
\hline
\end{tabular}

${ }^{*}$ Patients were all males with cyanotic heart conditions, ${ }^{* *} 6$ patient had multiple organ failure, 3 suffered from cardiovascular failure; tinclude hypoplastic right ventricle with VSD and ASD; VSD-ASD with patent ductus arteriosus; TGA without VSD

VSD - ventricular septal defect, TGA - transposition of great arteries, $A S D$ - atrial septal defect, DORV - double-outlet right ventricle

\section{Statistical analysis}

The data were analyzed using Statistical Package for Social Sciences (SPSS) version 14. A $p$ value of $<0.05$ was considered significant. Unpaired Student's $t$-test was used to assess the statistical significance of the difference between two population means in a study involving independent samples. The multiple regression test was used for assessing multiple linear regressions aiming to find a linear relationship between a response variable and several possible predictors. Correlation analysis was used for assessing the strength of association between two variables. The correlation coefficient ( $r$ ) defines the strength and direction of the linear relationship between two variables. Cut-off levels for sensitivity and specificity were determined using $\chi^{2}$; Fisher exact test was performed in tables containing a value less than 5 .

\section{Results}

The demographic data of the patients enrolled in the present study are shown in Table I. Patients were classified according to pediatric RIFLE criteria (2) into: $1-8$ patients (20\%) with no evidence or risk of AKI; $2-13$ (32.5\%) at risk for developing AKI (AKI grade I equivalent to RIFLE: Risk); 3 - 15 (37.5\%) showing evidence of AKI without failure (AKI grade II equivalent to RIFLE: Injury; and, 4 - 4 (10\%) suffering from acute renal failure (AKI grade III equivalent to RIFLE: Failure). For statistical reasons, the patients were divided into two groups: group I: patients who suffered AKI grades II and III (RIFLE: Injury and Failure); group II: patients who did not develop AKI or at risk (AKI grade I, RIFLE: Risk).

Comparison of serial plasma NGAL levels between patients of group I versus patients of group II is represented in Table II, and showed highly significant elevations in mean plasma NGAL levels in group I patients at 2, 12, and $24 \mathrm{~h}$ after surgery $(p<0.0001)$.

In Table III, significant correlations are shown between NGAL and creatinine at different time intervals, especially most evident at 12 and $24 \mathrm{~h}$ after surgery. Figure 1 shows the correlation coefficient $(r)$ of plasma NGAL versus serum creatinine with acute kidney injury at varying time intervals $(2,12$, and $24 \mathrm{~h}$ after surgery). Highly significant correlations $(p<0.0001)$ were recorded for plasma NGAL and AKI at 2, 12 and $24 \mathrm{~h}$ after surgery $(r=0.893, r=0.873$, and $r=0.795$ respectively). However, for serum creatinine, highly significant correlations with AKI were delayed and existed at 12 and $24 \mathrm{~h}$ post-operatively (0.609 and 0.711 respectively). Several cut-off levels were analyzed to explore the sensitivity and specificity of plasma NGAL to detect AKI grades II and III. A cut-off level of $100 \mathrm{ng} / \mathrm{ml} 2 \mathrm{~h}$ after surgery and $125 \mathrm{ng} / \mathrm{ml}$ at $12 \mathrm{~h}$ after surgery both recorded the highest accuracy, being 95\% accurate, with sensitivity of $100 \%$ and $89.5 \%$ respectively, and specificity of $90.5 \%$ and $100 \%$ respectively.

Our results show that sepsis was significantly evident in group I patients compared to group II $(p=0.034)$. Since it was observed that $72.5 \%$ of cases (29 patients) showed evidence of sepsis, diagnosed by an increase in total leukocytic count number associated by evident shift of band cells to the left during the first $48 \mathrm{~h}$ after surgery or by positive

Table II. Comparison of serial plasma NGAL levels between patients of group I versus patients of group II

\begin{tabular}{|lccccc|}
\hline Plasma NGAL [ng/dl] & \multicolumn{2}{c}{ Group I } & \multicolumn{2}{c|}{ Group II } & Value of $p$ \\
\cline { 2 - 5 } & \multicolumn{2}{c}{ AKI Grade II and III $(n=19)$} & No AKI and AKI grade I $(n=21)$ & SD \\
\cline { 2 - 5 } & Mean & SD & Mean & \pm 15.12 & 0.2483 \\
\hline Before surgery & 42.58 & \pm 16.51 & 36.17 & \pm 20.26 & $<0.0001^{*}$ \\
\hline 2 h after surgery & 154.79 & \pm 51.14 & 66.05 & \pm 21.59 & $<0.0001^{*}$ \\
\hline 12 h after surgery & 201.79 & \pm 54.89 & 83.67 & \pm 26.49 & $<0.0001^{*}$ \\
\hline 24 h after surgery & 238.74 & \pm 71.92 & 108.05 & & \\
\hline
\end{tabular}

*Highly significant difference; NGAL - neutrophil gelatinase associated lipocalin, AKI - acute kidney injury 
culture results [14], we further explored the relationship between sepsis and NGAL. After excluding cases with AKI grades II and III, we compared two groups: the first one showing evidence of sepsis versus the second group with no evidence of sepsis, as shown in Table IV. The difference between the groups was non-significant.

\section{Discussion}

Acute kidney injury, formerly known as acute renal failure, continues to represent a very common and potentially devastating problem in critically ill children $[15,16]$. Unfortunately, the mortality and morbidity associated with AKI remain unacceptably high, with mortality rates approaching $80 \%$ in critically ill children with multiple organ dysfunction syndrome [17].

The association between cardiopulmonary bypass and AKI is well recognized [18]. In current clinical practice, AKI is typically diagnosed by measuring an acute rise in serum creatinine. However, it is well known that creatinine is an unreliable and insensitive indicator during early, acute changes in kidney function, as serum creatinine concentrations typically do not change until approximately $50 \%$ of kidney function has already been lost [19].

In the present work, plasma NGAL mean levels were compared between group I (AKI grades II and III) and group II (no AKI and AKI grade I) (Table II). The results showed that there were highly significant differences in plasma NGAL levels between the groups; this significant difference started as early as $2 \mathrm{~h}$ after surgery $(p<0.0001)$, which reflects the potential role of plasma NGAL as an early biomarker in predicting AKI. The same results have been confirmed in many studies [12, 20, 21].

The relationship between plasma NGAL and serum creatinine levels, as well as the correlations of each marker with AKI at different time intervals, were studied (Table III, Figure 1).

Plasma NGAL levels were found to be significantly correlated with both serum creatinine and $\mathrm{AKI}$ at 2, 12 and $24 \mathrm{~h}$ after surgery, whereas serum creatinine did not show a significant correlation with AKI until after $12 \mathrm{~h}$ following surgery.
Table III. Correlation of plasma NGAL with serum creatinine at 2, 12 and $24 \mathrm{~h}$ after surgery

\begin{tabular}{|llc|}
\hline \multirow{2}{*}{ Serum creatinine $[\mathrm{mg} / \mathrm{dl}]$} & \multicolumn{2}{c|}{ Plasma NGAL $[\mathrm{ng} / \mathrm{dl}]$} \\
\cline { 2 - 3 } & Value of $r$ & Value of $p$ \\
\hline 2 h after surgery & 0.4758 & 0.0019 \\
\hline 12 h after surgery & 0.7194 & $<0.0001^{*}$ \\
\hline 24 h after surgery & 0.8075 & $<0.0001^{*}$ \\
\hline
\end{tabular}

*Highly significant correlation

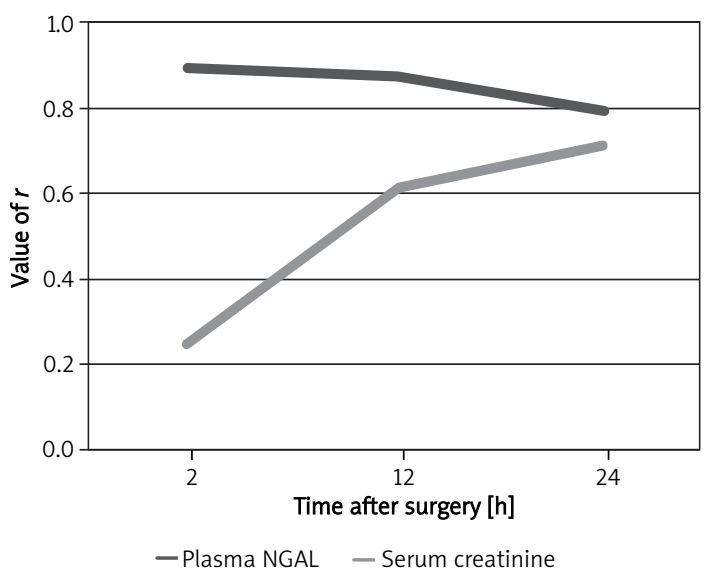

Figure 1. Correlation coefficient $(r)$ of plasma NGAL versus serum creatinine with acute kidney injury at varying time intervals (2, 12 , and $24 \mathrm{~h}$ after surgery) $N G A L$ - neutrophil gelatinase-associated lipocalin

Many studies have explored the relationship between NGAL and AKI in children and adults. Zappitelli et al. [12], in a study on critically ill pediatric patients, found that NGAL concentrations in AKI patients exhibited a six-fold increase in concentration that persisted from $48 \mathrm{~h}$ before to $48 \mathrm{~h}$ after development of AKI, and concluded that NGAL could serve well in predicting AKI before a rise in serum creatinine becomes apparent. In 2008, Devarajan [22] showed that plasma NGAL measurements could represent an early biomarker of AKI in the pediatric intensive care setting, being able to predict this complication about 2 days prior to the rise in serum creatinine with high sensitivity

Table IV. Plasma NGAL mean levels in patients with sepsis versus patients without sepsis after excluding cases with AKI grades II and III

\begin{tabular}{|lccccc|}
\hline Plasma NGAL [ng/dl] & \multicolumn{2}{c}{ Group I } & \multicolumn{2}{c|}{ Group II } & Value of $p$ \\
\cline { 2 - 6 } & \multicolumn{2}{c}{ Evidence of sepsis $(n=12)$} & No evidence of sepsis $(n=9)$ \\
\cline { 2 - 6 } & Mean & SD & Mean & SD & 0.3281 \\
\hline Before surgery & 39.58 & \pm 14.51 & 32.89 & \pm 15.92 & \pm .4530 \\
\hline 2 h after surgery & 63.08 & \pm 18.26 & 70 & \pm 23.18 & 0.3501 \\
\hline 12 h after surgery & 79.75 & \pm 16.36 & 88.89 & \pm 27.27 & \pm 24.97 \\
\hline 24 h after surgery & 102.33 & \pm 27.2 & 115.67 & & 0.2643 \\
\hline
\end{tabular}


and area under the curve (AUC) values of 0.68-0.78. The 2 -h post-operative plasma NGAL levels strongly correlated with the duration and severity of AKI and length of hospital stay.

Figure 1 shows the trend of both biomarkers in relation to AKI. Whereas plasma NGAL showed a rapid early rise $2 \mathrm{~h}$ after surgery which persisted until $12 \mathrm{~h}$ after surgery, with a slow decline thereafter, serum creatinine showed a slow rise which was non-significant at $2 \mathrm{~h}$ but started to be significant at $12 \mathrm{~h}$, with a further rise at $24 \mathrm{~h}$ after surgery.

These recorded results reflect the nature of both biomarkers. Regarding serum creatinine, it took time to build up and that is why it became significant as time passed. On the other hand, plasma NGAL pumped up its level early when AKI developed, which is why it was gradually washed out of the blood stream [23].

In order to explore the sensitivity and specificity of plasma NGAL level in predicting AKI grade II and III (RIFLE: Injury and Failure), several cut-off values were analyzed. Plasma NGAL level of $100 \mathrm{ng} / \mathrm{ml}$ at $2 \mathrm{~h}$ after surgery and $125 \mathrm{ng} / \mathrm{ml}$ at $12 \mathrm{~h}$ after surgery both recorded the highest accuracy, being 95\% accurate. The sensitivity and specificity of plasma NGAL cut-off value of $100 \mathrm{ng} / \mathrm{ml}$ at $2 \mathrm{~h}$ after surgery were $100 \%$ and $90.48 \%$ respectively, while the sensitivity and specificity of plasma NGAL cut-off value of $125 \mathrm{ng} / \mathrm{ml}$ at $12 \mathrm{~h}$ after surgery were $89.47 \%$ and $100 \%$ respectively.

The previous results show that plasma NGAL level could be used for detection of AKI as early as $2 \mathrm{~h}$ post-operatively and to confirm AKI at $12 \mathrm{~h}$ with great accuracy and high sensitivity and specificity.

Our results were close to a collective data analysis done in 2008 for some published performances of NGAL as a predictive biomarker for AKI, which revealed mean AUC-ROC of 0.82; the mean derived sensitivity and specificity from these studies were in the $75-80 \%$ range $[21,22]$. From the published studies, the derived optimal cut-off value of NGAL for the early prediction of AKI appeared to be at a median value of approximately $150 \mathrm{ng} / \mathrm{ml}$ [22].

Finally, the current study showed that sepsis was significantly evident in patients with AKI. Hence, we concluded that sepsis is a potential risk factor for developing AKI. Previous studies have mentioned that plasma NGAL levels might be affected by sepsis [24, 25]. In a study by Bagshaw et al. [26], critically ill patients with septic AKI were associated with significantly higher plasma NGAL at enrollment compared with non-septic AKI. However, in the present work, it seems that the levels of NGAL were not affected by sepsis as they showed non-significant differences between the group with sepsis versus the non-septic group after excluding the effect of overt AKI.

In conclusion, in this study plasma NGAL levels obtained at $2 \mathrm{~h}$ after surgery showed a highly sig- nificant correlation with $\mathrm{AKI}$, and this correlation remained high until $24 \mathrm{~h}$ after surgery. Hence, plasma NGAL could be used as an early biomarker for diagnosis of AKI following CPB in pediatric cardiac surgery. We recommend further studies on a wider scale to validate the current study results.

\section{References}

1. Mehta RL, Kellum JA, Shah SV, et al. Acute kidney network: report of an initiative to improve outcomes in acute kidney injury. Crit Care 2007; 11: 1-8.

2. Bellomo R, Ronco C, Kellum JA, Mehta RL, Palevsky P. Acute dialysis quality Initiative workgroup. Acute renal failure - definition, outcome measures, animal models, fluid therapy and information technology needs: the Second International Consensus Conference of the Acute Dialysis Quality Initiative (ADQI) Group. Crit Care 2004; 8: R204-12.

3. Akcan-Arikan A, Zappitelli M, Loftis LL, Washburn KK, Jefferson LS, Goldstein SL. Modified RIFLE criteria in critically ill children with acute kidney injury. Kidney Int 2007; 71: 1028-35.

4. Liangos O, Wald R, O’Bell JW, Price L, Pereira BJ, Jaber BL. Epidemiology and outcomes of acute renal failure in hospitalized patients: a national survey. Clin J Am Soc Nephrol 2006; 1: 43-51.

5. Palevsky PM. Epidemiology of acute renal failure: the tip of the iceberg. Clin J Am Soc Nephrol 2006; 1: 6-7.

6. Albert MA, Antman EM. Preoperative evaluation for cardiac surgery. In: Cardiac surgery in the adult. Cohn LH, Edmunds LH Jr (eds). McGraw-Hill, New York, 2003; 235-48.

7. Haase M, Haase-Fielitz A, Bagshaw SM, Ronco C, Bellomo R. Cardiopulmonary bypass-asociated acute kidney injury: a pigment nephropathy? Contrib Nephrol 2007; 156: 340-53.

8. Chertow GM, Levy EM, Hammermesiter KE, Grover F, Daley J. Independent association between acute renal failure and mortality following cardiac surgery. Am J Med 1998; 104: 343-8.

9. Hsu CW, Symons JM. Acute kidney injury: can we improve prognosis? Pediatr Nephrol 2010; 12: 2401-12.

10. Boer DP, de Rijke YB, Hop WC, Cransberg K, Dorresteijn EM. Reference values for serum creatinine in children younger than 1 year of age. Pediatr Nephrol 2010; 25: 2107-13.

11. Schmidt-Ott KM, Mori K, Kalandadze A, et al. Neutrophil gelatinase-associated lipocalin-mediated iron traffic in kidney epithelia. Curr Opin Nephrol Hypertens 2006; 15: 442-9.

12. Zappitelli M, Washburn KK, Arikan AA, et al. Urine neutrophil gelatinase-associated lipocalin is an early marker of acute kidney injury in critically ill children: a prospective cohort study. Crit Care 2007; 11: R84.

13. Mcllory RD, Wagener G, Lee TH. Evolving biomarkers for acute kidney injury. Anesthesiology 2010; 112: 998-1004.

14. Levy MM, Fink MP, Marshall JC, et al. 2001 SCCM/ESICM/ ACCP/ATS/SIS International Sepsis Definitions Conference. Crit Care Med 2003; 31: 1250-6.

15. Hui-Stickle S, Brewer ED, Goldstein SL. Pediatric ARF epidemiology at a tertiary care center from 1999 to 2001. Am J Kidney Dis 2005; 45: 96-101.

16. Uchino S. The epidemiology of acute renal failure in the world. Curr Opin Crit Care 2006; 12: 538-43.

17. Sutherland SM, Zappitelli M, Alexander SR, et al. Fluid overload and mortality in children receiving continuous 
renal replacement therapy: the prospective pediatric continuous renal replacement therapy registry. Am J Kidney Dis 2010; 55: 316-25.

18. Abu-Omar Y, Ratnatunga C. Cardiopulmonary bypass and renal injury. Perfusion 2006; 21: 209-13.

19. Moran SM, Myers BD. Course of acute renal failure studied by a model of creatinine kinetics. Kidney Int 1985; 27: 928-37.

20. Mishra J, Dent C, Tarabishi R, et al. Neutrophil gelatinase associated lipocalin (NGAL) as a biomarker for acute renal injury after cardiac surgery. Lancet 2005; 365: 1231-8.

21. Devarajan P. Neutrophil gelatinase-associated lipocalin (NGAL): a new marker of kidney disease. Scand J Clin Lab Invest Suppl 2008; 241: 89-94.

22. Devarajan P. Neutrophil gelatinase associated lipocalinan emerging troponin for kidney injury. Nephrol Dial Transplant 2008; 23: 3737-43.

23. Dent $\mathrm{CL}, \mathrm{Ma} \mathrm{Q}$, Dastrala $\mathrm{S}$, et al. Plasma neutrophil gelatinase-associated lipocalin predicts acute kidney injury, morbidity and mortality after pediatric cardiac surgery: a prospective uncontrolled cohort study. Crit Care 2007; 11: R127.

24. Xu SY, Pauksen K, Venge P. Serum measurements of human neutrophil lipocalin (HNL) discriminate between acute bacterial and viral infections. Scand J Clin Lab Invest 1995; 55: 125-31.

25. Wheeler DS, Devarajan P, Ma Q, et al. Serum neutrophil gelatinase-associated lipocalin (NGAL) as a marker of acute kidney injury in critically ill children with septic shock. Crit Care Med 2008; 36: 1297-303.

26. Bagshaw SM, Bennett M, Haase $M$, et al. Plasma and urine neutrophil gelatinase-associated lipocalin in septic versus non-septic acute kidney injury in critical illness. Int Care Med 2010; 36: 452-61. 